\title{
Correction to "6-Mercaptopurine Decreases the Bcl-2/Bax Ratio and Induces Apoptosis in Activated Splenic B Lymphocytes"
}

In the above article [Hortelano S and Boscá L (1997) Mol Pharmacol 51:414-421], the authors responded to concerns regarding Figure 2 and provided the uncropped image, which was subsequently analyzed by the journal. In this figure, one lane was removed from the original image because it was not relevant. All lanes were from the same gel, and there was no duplication of lanes. The legend has been edited below to indicate that this is the case. The results were not affected.

Legend: 6-MP promotes apoptosis in activated B cells. Purified small B lymphocytes $\left(1 \times 10^{6}\right)$ were incubated for $8 \mathrm{hr}$ in the absence (-) or presence (+) of $20 \mu \mathrm{M} 6$-MP and the indicated mitogens ( $2 \mu \mathrm{g} / \mathrm{ml}$ LPS and $10 \mu \mathrm{g} / \mathrm{ml}$ anti-CD40 mAb). After this incubation period, the cells were centrifuged and homogenized; after isolation, the DNA was analyzed in a $1 \%$ agarose gel. Results show the analysis of the indicated lanes from a representative gel (out of four independent experiments) in which bands corresponding to other conditions between the 6-MP and the 6-MP+LPS+anti-CD40 mAb lanes were removed.

The authors regret this error and any inconvenience it may have caused. 